EGU2020-18452

https://doi.org/10.5194/egusphere-egu2020-18452

EGU General Assembly 2020

(c) Author(s) 2021. This work is distributed under

the Creative Commons Attribution 4.0 License.

\title{
Measuring bedload transport rates in a laboratory flume: fluctuations and uncertainties
}

\author{
Ivan Pascal and Christophe Ancey \\ Environmental Hydraulics Laboratory, EPFL, Lausanne, Switzerland (ivan.pascal@epfl.ch, christophe.ancey@epfl.ch)
}

Measuring sediment fluxes in rivers and laboratory flumes has long been a challenge. Different definitions of sediment transport rates have been proposed over the past decades. Most measurement techniques involve collecting a volume of sediment in a sampler or counting the number of particles crossing a reference section within a given time interval. In laboratory experiments, scientists routinely use high-speed cameras and particle tracking techniques for monitoring bedload transport, but measuring the relevant transport parameters (i.e. the number of moving particles, their velocities and size, etc.) remains a demanding task. Moreover, no clear consensus has emerged on how to define mean bedload transport rates. To address this controversy, we ran an experiment in which we measured the particle flux in different places along a flume using high-speed cameras. Furthermore, we also determined the number of particles moving in a fixed control volume, their trajectories, and their velocities. Even under steady-state conditions, particle transport rates exhibited significant non-Gaussian fluctuations, which caused the time-averaged transport rate to fluctuate widely. In such a situation, determining the mean transport rate becomes a non-trivial operation. To solve this issue, we developed a procedure for estimating the uncertainties associated with the time-averaged transport rates. The theoretical underpinnings are provided by a Markovian model of bedload transport. We demonstrated its versatility by applying it to other laboratory and field cases with different monitoring systems. 